\title{
On the relation between turnpike properties for finite and infinite horizon optimal control problems
}

\author{
Lars Grüne* \\ Mathematical Institute \\ University of Bayreuth \\ 95440 Bayreuth, Germany \\ lars.gruene@uni-bayreuth.de \\ Christopher M. Kellett, Steven R. Weller \\ School of Electrical Engineering and Computer Science \\ University of Newcastle \\ Callaghan, NSW 2308, Australia \\ chris.kellett, steven.weller@newcastle.edu.au
}

October 27, 2016

\begin{abstract}
We show that under appropriate regularity conditions a finite horizon optimal control problem exhibits the turnpike property if and only if its infinite horizon counterpart does. We prove the result for undiscounted and for discounted problems and also provide a version which incorporates quantitative information about the convergence rates.
\end{abstract}

Keywords: finite horizon optimal control, infinite horizon optimal control, optimal equilibrium

\section{Introduction}

The turnpike phenomenon is a property of trajectories of optimally controlled systems that has long been observed in optimal control, even back to early work by von Neumann [13]. The turnpike property describes the fact that an optimal trajectory "most of the time" stays close to an equilibrium point, as illustrated in Figure 3.1, below, for finite horizon optimal trajectories. This property attracted significant interest, particularly in the field of mathematical economics [10], because it directly leads to the concept of optimal economic equilibria and thus provides a natural economic interpretation of optimality. The name "turnpike property" was coined in 1958 in the book by Dorfman, Samuelson and Solow [4], who compared the phenomenon to the optimal way of driving by car from a point A to a point B using a turnpike or highway, which consists of three phases: driving to the

\footnotetext{
${ }^{*}$ C. M. Kellett and L. Grüne are supported by the Australian Research Council under DP160102138. L. Grüne is supported by the Deutsche Forschungsgemeinschaft, Grant GR 1569/13-1. The research for this paper was carried out while L. Grüne was visiting the University of Newcastle, Australia
} 
highway (i.e., approaching the equilibrium), driving on the highway (i.e., staying near the equilibrium) and leaving the highway (i.e., moving away from the equilibrium).

Recently, the turnpike property has also attracted interest in areas different from mathematical economics, see, e.g., $[5,14,12,8]$. This interest stems from the fact that it was realized that this property considerably simplifies the computation of (approximately) optimal trajectories in all areas of optimal control, either directly by constructive synthesis techniques as in [1] or indirectly via a receding horizon approach as in economic model predictive control $[6,7] .{ }^{1}$ Moreover, the turnpike property can also be rigorously established in control systems governed by partial differential equations [11], significantly enlarging the classes of systems for which these methods are applicable. In the context of economic model predictive control, strict dissipativity can be used as an alternative to assuming the turnpike property [2]. However, as [8] shows, these two properties are almost equivalent, in the sense that under a controllability and a reachability condition, strict dissipativity holds if and only if a robust version of the turnpike property holds. "Robust" here refers to the fact that not only optimal but also approximately optimal trajectories exhibit the turnpike phenomenon, though in a relaxed form. This robust version of the turnpike property has additional structure, making it more suitable for rigorous mathematical proofs, which is why we also use it in this paper. We will, however, use neither strict dissipativity nor controllability assumptions in this paper.

Many of the papers and books discussed above provide sufficient (and sometimes also necessary) conditions for the occurrence of turnpike behavior. However, all results we are aware of apply to either finite horizon or infinite horizon optimal control problems. In contrast to this, in this paper we study the relation between turnpike properties for these two classes of optimal control problems. More precisely, we show that under suitable regularity conditions, the turnpike phenomenon occurs in the finite horizon problem if and only if it occurs in the infinite horizon problem with the same stage cost. The study is carried out for discrete time systems, mainly because this somewhat reduces the analytic overhead and simplifies some arguments. However, we expect that a similar reasoning is also possible in the continuous time setting.

The paper is organized as follows. In Section 2 we describe the optimal control problem we are considering. In Section 3 we show the relation between the finite and the infinite horizon property for undiscounted problems. In Section 4 we repeat the analysis for discounted problems since these problems require significantly different assumptions and proof techniques. In Section 5 we discuss turnpike properties which also include quantitative bounds on the convergence rate of the trajectories to the equilibrium. Section 6 concludes the paper.

\section{Setting and preliminaries}

We consider possibly discounted discrete time optimal control problems

$$
\underset{u \in \mathbb{U}^{N}\left(x_{0}\right)}{\operatorname{minimize}} J_{N}\left(x_{0}, u\right)
$$

\footnotetext{
${ }^{1}$ Despite its name, economic model predictive control was developed in control engineering rather than in mathematical economics.
} 
where

$$
\begin{gathered}
J_{N}\left(x_{0}, u\right):=\sum_{k=0}^{N-1} \beta^{k} \ell(x(k), u(k)), \\
x(n+1)=f(x(n), u(n)), \quad x(0)=x_{0},
\end{gathered}
$$

$N \in \mathbb{N}$ or $N=\infty, \beta \in(0,1], f: X \times U \rightarrow X$ for metric spaces $X$ and $U$, state and input constraints $\mathbb{X} \subseteq X, \mathbb{U} \subseteq U$ and admissible control sets $\mathbb{U}^{N}\left(x_{0}\right):=\left\{u(\cdot) \in U^{N} \mid x(n) \in\right.$ $\mathbb{X} \forall n=0, \ldots, N$ and $u(n) \in \mathbb{U} \forall n=0, \ldots, N-1\}$. In what follows, for simplicity of exposition we assume $\mathbb{U}^{N}\left(x_{0}\right) \neq \emptyset$ for all $x_{0} \in \mathbb{X}$ and all $N \in \mathbb{N}$ (which implies $\mathbb{U}^{\infty}\left(x_{0}\right) \neq \emptyset$ ). If this is not the case, all results remain true if we restrict ourselves to initial conditions $x_{0}$ for which $\mathbb{U}^{\infty}\left(x_{0}\right) \neq \emptyset$ and to control functions from $\mathbb{U}^{\infty}\left(x_{0}\right)$. We define the optimal value function

$$
V_{N}\left(x_{0}\right):=\inf _{u \in \mathbb{U}^{N}\left(x_{0}\right)} J_{N}\left(x_{0}, u\right)
$$

As we will see, both the proofs and the assumptions on the problem differ considerably between the undiscounted case $\beta=1$ and the discounted case $\beta \in(0,1)$. We therefore treat these two cases in two separate sections and start with the undiscounted case. In what follows, we denote the cardinality of a set $S \subset \mathbb{N}$ by $\# S$.

\section{The undiscounted case}

In this section we consider the undiscounted case, i.e., the case $\beta=1$. We first define the precise meaning of the turnpike properties under consideration.

Definition 3.1: (finite horizon turnpike property) The optimal control problem (2.1) has the finite horizon robust turnpike property at an equilibrium $x^{e} \in \mathbb{X}$, if for each $\delta>0$, each $\varepsilon>0$ and each bounded set $\mathbb{X}_{b} \subset \mathbb{X}$ there is a constant $C_{\delta, \varepsilon, \mathbb{X}_{b}}^{f i n} \in \mathbb{N}$ such that all trajectories $(x(k), u(k))$ with $x_{0} \in \mathbb{X}_{b}, u(\cdot) \in \mathbb{U}^{N}\left(x_{0}\right)$ and arbitrary $N \in \mathbb{N}$ satisfying $J_{N}\left(x_{0}, u\right) \leq V_{N}\left(x_{0}\right)+\delta$ satisfy

$$
\#\left\{k \in\{0, \ldots, N\} \mid d\left(x(k), x^{e}\right) \geq \varepsilon\right\} \leq C_{\delta, \varepsilon, \mathbb{X}_{b}}^{f i n} .
$$

In words, this definition demands that given $\varepsilon>0$ and $\delta>0$, for every $\delta$-optimal trajectory starting in $\mathbb{X}_{b}$, all but at most $C_{\delta, \varepsilon, \mathbb{X}_{b}}^{f i n}$ points on the trajectory lie in an $\varepsilon$-neighborhood of $x^{e}$. The important property of the constant $C_{\delta, \varepsilon, \mathbb{X}_{b}}^{f i n}$ is that it does not depend on $N$, i.e., the bound on the number of points outside of the $\varepsilon$-neighborhood of $x^{e}$ is independent of $N$. Figure 3.1 shows finite horizon optimal trajectories on different horizons $N$ which exhibit the turnpike property. For the details of the optimal control problems behind these figures we refer to [7].

Definition 3.2: (infinite horizon turnpike property) The optimal control problem (2.1) has the infinite horizon robust turnpike property at an equilibrium $x^{e} \in \mathbb{X}$, if for each $\delta>0$, each $\varepsilon>0$ and each bounded set $\mathbb{X}_{b} \subset \mathbb{X}$ there is a constant $C_{\delta, \varepsilon, \mathbb{X}_{b}}^{\infty} \in \mathbb{N}$ such that 

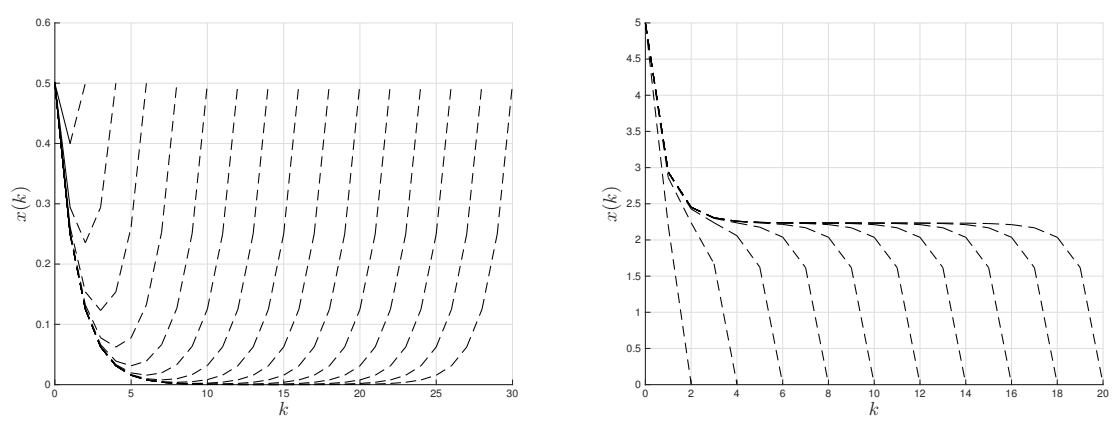

Figure 3.1: Finite horizon optimal trajectories $x(\cdot)$ (dashed) for different optimization horizons $N=2,4, \ldots, 30$ (left) and $N=2,4, \ldots, 20$ (right) for two examples from [7]

all trajectories $(x(k), u(k))$ with $x_{0} \in \mathbb{X}_{b}, u(\cdot) \in \mathbb{U}^{\infty}\left(x_{0}\right)$ satisfying $J_{\infty}\left(x_{0}, u\right) \leq V_{\infty}\left(x_{0}\right)+\delta$ satisfy

$$
\#\left\{k \in \mathbb{N} \mid d\left(x(k), x^{e}\right) \geq \varepsilon\right\} \leq C_{\delta, \varepsilon, \mathbb{X}_{b}}^{\infty} .
$$

Note that Definition 3.2 implies $\lim _{k \rightarrow \infty} d\left(x(k), x^{e}\right)=0$, because if this convergence does not hold, then there exists $\varepsilon>0$ and a sequence $k_{j} \rightarrow \infty$ with $d\left(x\left(k_{j}\right), x^{e}\right) \geq \varepsilon$ for all $j \in \mathbb{N}$. This however, implies $\#\left\{k \in \mathbb{N} \mid d\left(x(k), x^{e}\right) \geq \varepsilon\right\}=\infty$, and hence the property from Definition 3.2 cannot hold. Therefore, the infinite horizon turnpike property implies convergence of the respective trajectories to the equilibrium $x^{e}$. However, the rate of convergence can be arbitrarily slow, since we do not make any assumption about the size of the time instant $k$ in $\#\left\{k \in \mathbb{N} \mid d\left(x(k), x^{e}\right) \geq \varepsilon\right\}$. We will address this issue in Section 5 .

In order to establish a relation between Definitions 3.1 and 3.2, we make the following regularity assumptions on the problem.

Assumption 3.3: We assume the following for optimal control problem (2.1).

(i) For each bounded subset $\mathbb{X}_{b} \subseteq \mathbb{X}$ there exists $C>0$ such that $\left|V_{N}(x)\right| \leq C$ holds for all $x \in \mathbb{X}_{b}$ and all $N \in \mathbb{N} \cup\{\infty\}$.

(ii) for each $\Theta>0$ there is a bounded set $\mathbb{X}_{\Theta} \subseteq \mathbb{X}$ such that for each $N \in \mathbb{N} \cup\{\infty\}$ the inequality $J_{N}\left(x_{0}, u\right) \leq \Theta$ implies $x(k) \in \mathbb{X}_{\Theta}$ for all $k=0, \ldots, N$.

Part (i) of Assumption 3.3 is a boundedness condition which demands that the optimal value functions are uniformly (w.r.t. $N$ and including $N=\infty$ ) bounded on bounded sets, both from above and from below. It is needed to rule out degenerate behavior caused by unbounded accumulated cost.

Part (ii) effectively states that trajectories with bounded values stay in bounded sets. There are (at least) two easy ways to ensure that this condition holds: on the one hand, we may assume that $\mathbb{X}$ itself is bounded, in which case we can always choose $\mathbb{X}_{\Theta}=\mathbb{X}$. 
Alternatively, we may assume the existence of constants $C_{1}, C_{2}, C_{3} \in \mathbb{R}$ with $C_{2}>0$ and a point $\hat{x} \in \mathbb{X}$ such that $\ell(x, u) \geq C_{1}+C_{2} d(x, \hat{x})$ and $V_{N}(x) \geq C_{3}$ holds for all $x \in \mathbb{X}$, all $u \in \mathbb{U}$ and all $N \in \mathbb{N} \cup\{\infty\}$. In this case, the existence of $k \in \mathbb{N}$ with $k \leq N$ with $d(x(k), \hat{x})>\Delta$ implies

$J_{N}(x, u)=J_{k}(x, u)+\ell(x(k), u(k))+J_{N-k-1}(x(k+1), u(\cdot+k+1))>C_{3}+C_{1}+C_{2} \Delta+C_{3}$,

hence $J_{N}(x, u) \leq \Theta$ implies $\Delta \leq\left(\Theta-C_{1}-2 C_{3}\right) / C_{2}$, and thus $\mathbb{X}_{\Theta}$ can be chosen as the closed ball with radius $\left(\Theta-C_{1}-2 C_{3}\right) / C_{2}$ around $\hat{x}$.

The following theorem now gives the main result of this section.

Theorem 3.4: Consider the optimal control problem (2.1) satisfying Assumption 3.3. Then the finite horizon turnpike property from Definition 3.1 holds if and only if the infinite horizon turnpike property from Definition 3.2 holds.

Proof. "Definition $3.1 \Rightarrow$ Definition 3.2": Assume that the problem has the finite horizon turnpike property from Definition 3.1. We show that the problem then also has the infinite horizon turnpike property from Definition 3.2. To this end, we consider a trajectory satisfying the conditions from Definition 3.2. That is, we pick $\delta>0, \varepsilon>0$, a bounded subset $\mathbb{X}_{b} \subseteq \mathbb{X}$ and an infinite trajectory with $x_{0} \in \mathbb{X}_{b}$ satisfying $J_{\infty}\left(x_{0}, u\right) \leq V_{\infty}\left(x_{0}\right)+\delta$.

Next we verify that the trajectory also satisfies the conditions from Definition 3.1. For this purpose, let $C$ denote the bound from Assumption 3.3(i), which implies $\left|V_{N}(x)-V_{\infty}(x)\right| \leq$ $K=2 C$ for $x \in \mathbb{X}_{b}$. Then Assumption 3.3(ii) implies that $x(k) \in \mathbb{X}_{\Theta}$ for all $k \in \mathbb{N}$ and a bounded set $\mathbb{X}_{\Theta}$ with $\Theta=K+\delta$, which by Assumption 3.3(i) yields the existence of $\widetilde{K}>0$ with $V_{\infty}(x(k)) \geq-\widetilde{K}$ for all $k \in \mathbb{N}$. For all $N \in \mathbb{N}$ we have

$$
V_{\infty}\left(x_{0}\right)+\delta \geq J_{\infty}\left(x_{0}, u\right)=J_{N}\left(x_{0}, u\right)+\beta^{N} J_{\infty}(x(N), u(N+\cdot)) \geq J_{N}\left(x_{0}, u\right)-\beta^{N} \widetilde{K}
$$

implying

$$
J_{N}\left(x_{0}, u\right) \leq V_{\infty}\left(x_{0}\right)+\delta+\beta^{N} \widetilde{K} \leq V_{N}\left(x_{0}\right)+K+\delta+\widetilde{K} .
$$

Thus, the conditions from Definition 3.1 are satisfied, and since by assumption the problem has the finite horizon robust turnpike property, from (3.1) we obtain

$$
\#\left\{k \in\{0, \ldots, N\} \mid d\left(x(k), x^{e}\right) \geq \varepsilon\right\} \leq C_{\delta+K+\widetilde{K}, \varepsilon, \mathbb{X}_{b}}^{f i n}
$$

for all $N \in \mathbb{N}$, which implies (3.2) with

$$
C_{\delta, \varepsilon, \mathbb{X}_{b}}^{\infty}=C_{\delta+K+\widetilde{K}, \varepsilon, \mathbb{X}_{b}}^{f i n},
$$

and thus the infinite horizon robust turnpike property according to Definition 3.2.

"Definition $3.2 \Rightarrow$ Definition 3.1": We proceed similarly as above for the converse direction and consider a trajectory satisfying the conditions from Definition 3.1. To this end, fix $\delta>0, \varepsilon>0, N \in \mathbb{N}$ a bounded subset $\mathbb{X}_{b} \subseteq \mathbb{X}$ and a trajectory of length $N$ with $x_{0} \in \mathbb{X}_{b}$ satisfying $J_{N}\left(x_{0}, u\right) \leq V_{N}\left(x_{0}\right)+\delta$.

Now we construct an extended trajectory which satisfies the conditions from Definition 3.1: letting $K=2 C$ denote the bound on the difference $\left|V_{N}(x)-V_{\infty}(x)\right|$ from Assumption 3.3(i), by Assumption 3.3(ii) we can conclude the existence of a bounded set $\mathbb{X}_{\Theta}$ with 
$x(N) \in \mathbb{X}_{\Theta}$ and, hence, again by Assumption 3.3(i) of a constant $\widetilde{K}$ with $V_{\infty}(x(N)) \leq \widetilde{K}$. Picking a control function $\tilde{u}$ satisfying $J_{\infty}(x(N), \tilde{u}) \leq \widetilde{K}+\delta$ and defining

$$
\hat{u}(k):=\left\{\begin{array}{ll}
u(k), & k=0, \ldots, N-1 \\
\tilde{u}(k-N), & k=N, N+1, \ldots
\end{array},\right.
$$

we thus obtain

$$
J_{\infty}\left(x_{0}, \hat{u}\right)=J_{N}\left(x_{0}, u\right)+\beta^{N} J_{\infty}(x(N), \tilde{u}) \leq V_{N}\left(x_{0}\right)+\delta+\widetilde{K}+\delta \leq V_{\infty}\left(x_{0}\right)+K+\widetilde{K}+2 \delta .
$$

Hence, by (3.2) the extended trajectory satisfies

$$
\#\left\{k \in \mathbb{N} \mid d\left(x(k), x^{e}\right) \geq \varepsilon\right\} \leq C_{2 \delta+K+\widetilde{K}, \varepsilon, \mathbb{X}_{b}}^{\infty} .
$$

which implies the finite horizon turnpike property (3.1) with $C_{\delta, \varepsilon, \mathbb{X}_{b}}^{f i n}=C_{2 \delta+K+\widetilde{K}, \varepsilon, \mathbb{X}_{b}}^{\infty}$.

\section{The discounted case}

We now turn our attention to the discounted case with $\beta \in(0,1)$. For our analysis, the decisive difference in the discounted case is that the discount factor $\beta^{k}$ tends to 0 as $k$ tends to infinity. This means that if a trajectory has a large deviation from the optimal trajectory, then this large deviation may nevertheless be barely visible in the cost functional, provided it happens sufficiently late. For this reason, it is unreasonable to expect that one can see the turnpike behavior for trajectories satisfying $J_{N}(x, u) \leq V_{N}(x)+\delta$. In order to fix this problem, we need make two changes to the robust turnpike Definitions 3.1 and 3.2. First, we need to restrict the time interval on which we can expect to see the turnpike phenomenon and second, we need to limit the difference $\delta$ between the value of the trajectory under consideration and the optimal value. In the following definitions, the first will be taken care of by introducing the discrete time interval $\{0, \ldots, M\}$ and the second by defining the bound $\delta_{\varepsilon, M, \mathbb{X}_{b}}^{\text {fin }}$.

Definition 4.1: (finite horizon turnpike property) The optimal control problem (2.1) has the finite horizon near optimal approximate turnpike property, if for each $\varepsilon>0$ and each bounded set $\mathbb{X}_{b} \subset \mathbb{X}$ there is a constant $C_{\varepsilon, \mathbb{X}_{b}}^{\text {fin }}>0$ such that for each $M \in \mathbb{N}$ there is a constant $\delta=\delta_{\varepsilon, M, \mathbb{X}_{b}}^{\text {fin }}>0$ such that for all $N \in \mathbb{N}$ with $N \geq M$, all trajectories $(x(k), u(k))$ with $x_{0} \in \mathbb{X}_{b}, u(\cdot) \in \mathbb{U}^{N}\left(x_{0}\right)$ and $J_{N}\left(x_{0}, u\right) \leq V_{N}\left(x_{0}\right)+\delta$ satisfy

$$
\#\left\{k \in\{0, \ldots, M\} \mid d\left(x(k), x^{e}\right) \geq \varepsilon\right\} \leq C_{\varepsilon, \mathbb{X}_{b}}^{\text {fin }} .
$$

Definition 4.2: (infinite horizon turnpike property) The optimal control problem (2.1) has the infinite horizon near optimal approximate turnpike property, if for each $\varepsilon>0$ and each bounded set $\mathbb{X}_{b} \subset \mathbb{X}$ there is a constant $C_{\varepsilon, \mathbb{X}_{b}}^{\infty}>0$ such that for each $M \in \mathbb{N}$ there is a constant $\delta=\delta_{\varepsilon, M, \mathbb{X}_{b}}^{\infty}>0$ such that all trajectories $(x(k), u(k))$ with $x_{0} \in \mathbb{X}_{b}$, $u(\cdot) \in \mathbb{U}^{\infty}\left(x_{0}\right)$ and $J_{\infty}\left(x_{0}, u\right) \leq V_{\infty}\left(x_{0}\right)+\delta$ satisfy

$$
\#\left\{k \in\{0, \ldots, M\} \mid d\left(x(k), x^{e}\right) \geq \varepsilon\right\} \leq C_{\varepsilon, \mathbb{X}_{b}}^{\infty} .
$$


We note that in both definitions the level $\delta$ which measures the deviation from optimality depends on $M$. In both definitions, $\delta \rightarrow 0$ may be required if $M \rightarrow \infty$. It is, however, easily seen that the definitions imply (3.2) for the optimal trajectories (i.e., for $\delta=0$ ),

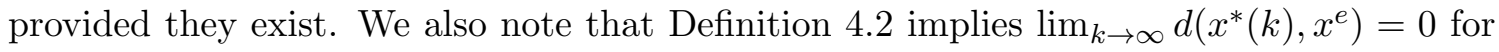
the optimal trajectory, again provided it exists.

Similar to the definitions of the turnpike property, we also need to adapt Assumption 3.3 to the discounted case.

Assumption 4.3: We assume the following for optimal control problem (2.1).

(i) $V_{N} \rightarrow V_{\infty}$ as $N \rightarrow \infty$ uniformly on bounded subsets of $\mathbb{X}$.

(ii) For each $\tilde{\varepsilon}>0$ and each bounded set $\mathbb{X}_{b} \subseteq \mathbb{X}$ there is $N_{0} \in \mathbb{N}$ with the following property: for each $N^{\prime} \geq N_{0}$ there is $\tilde{\delta}>0$ such that for all $x_{0} \in \mathbb{X}_{b}$, all $N \in \mathbb{N} \cup\{\infty\}$ with $N \geq N^{\prime}$ and all $u \in \mathbb{U}^{N}\left(x_{0}\right)$ satisfying the inequality $J_{N}\left(x_{0}, u\right) \leq V_{N}\left(x_{0}\right)+\tilde{\delta}$, the inequality $\beta^{N^{\prime}}\left|V_{N^{\prime \prime}}\left(x\left(N^{\prime}\right)\right)\right| \leq \tilde{\varepsilon}$ holds for all $N^{\prime \prime} \in \mathbb{N} \cup\{\infty\}$.

Assumption 4.3(i) states that the two operations "taking the infimum of $J_{N}(x, u)$ w.r.t. $u$ " and "passing to the limit for $N \rightarrow \infty$ " can be interchanged without changing the value. While this would be a rather strong assumption for undiscounted problems, for discounted problems it is always satisfied if, e.g., the stage cost is bounded along the optimal trajectories. In this case, due to the exponential decay of $\beta^{k}$, the value of a tail of an optimal trajectory becomes arbitrarily small, and hence also the difference between minimizing $J_{N}$ and $J_{\infty}$ becomes arbitrarily small. Therefore, Assumption 4.3(i) is always satisfied if, e.g., $\ell$ is bounded on $\mathbb{X}$ or at least on a set containing the optimal trajectories starting in a bounded set.

Assumption 4.3(ii) is relatively technical, but, again, since $\beta^{k} \rightarrow 0$ as $k \rightarrow \infty$, if we know that the modulus of the optimal value functions $\left|V_{N}\right|, N \in \mathbb{N} \cup\{\infty\}$ is bounded along the trajectories $x(\cdot)$, say by a constant $C$, then it suffices to choose $N^{\prime}$ so large that $\beta^{N^{\prime}} C \leq \tilde{\varepsilon}$ holds. Again, this boundedness holds, e.g., if the $\left|V_{N}\right|$ are uniformly bounded on the whole set $\mathbb{X}$ or if they are bounded on bounded sets and the near optimal trajectories $x(\cdot)$ stay in bounded sets up to the time $N^{\prime}$. Since the last two properties are implied by Assumption 3.3(i) and (ii), Part (ii) of Assumption 4.3 can be seen as a relaxation of Assumption 3.3.

The counterpart of Theorem 3.4 for the discounted case now reads as follows.

Theorem 4.4: Consider the optimal control problem (2.1) satisfying Assumption 4.3. Then the finite horizon turnpike property from Definition 4.1 holds if and only if the infinite horizon turnpike property from Definition 4.2 holds.

Proof. "Definition $4.1 \Rightarrow$ Definition 4.2": Similar to the first part of the proof of Theorem 3.4 we consider a trajectory satisfying the conditions of Definition 4.2 and show that it also satisfies the conditions of Definition 4.1, from which we then conclude (4.2). However, now we need some preliminary considerations in order to determine the bound on $\delta$ in Definition 4.2. To this end, fix $\varepsilon>0$, a bounded set $\mathbb{X}_{b} \subseteq \mathbb{X}$ and $M \in \mathbb{N}$ and let $\delta^{f i n}=\delta_{\varepsilon, M, \mathbb{X}_{b}}^{f i n}>0$ be the level of accuracy needed in Definition 4.1. We set $\tilde{\delta}:=\delta^{f i n} / 4$, pick $N_{0} \in \mathbb{N}$ from Assumption 4.3(ii) and from Assumption 4.3(i) we choose $N \geq \max \left\{N_{0}, M\right\}$ so large that $\left|V_{N}\left(x_{0}\right)-V_{\infty}\left(x_{0}\right)\right| \leq \tilde{\delta}$ for all $x_{0} \in \mathbb{X}_{b}$. For this $N$, we take $\tilde{\varepsilon}>0$ from 
Assumption $4.3\left(\right.$ ii) and set $\delta:=\min \left\{\tilde{\varepsilon}, \delta^{f i n} / 2\right\}$. Now we consider a trajectory satisfying $J_{\infty}\left(x_{0}, u\right) \leq V_{\infty}\left(x_{0}\right)+\delta$.

Then, using Assumption 4.3(ii) with $N=N^{\prime}$ and $N^{\prime \prime}=\infty$ we obtain

$$
\begin{aligned}
V_{N}\left(x_{0}\right)+\delta+\tilde{\delta} & \geq V_{\infty}\left(x_{0}\right)+\delta \geq J_{\infty}\left(x_{0}, u\right)=J_{N}\left(x_{0}, u\right)+\beta^{N} J_{\infty}(x(N), u(N+\cdot)) \\
& \geq J_{N}\left(x_{0}, u\right)+\beta^{N} V_{\infty}(x(N)) \geq J_{N}\left(x_{0}, u\right)-\tilde{\delta}
\end{aligned}
$$

i.e.,

$$
J_{N}\left(x_{0}, u\right) \leq V_{N}\left(x_{0}\right)+\delta+2 \tilde{\delta} \leq V_{N}\left(x_{0}\right)+\delta^{\text {fin }}
$$

This implies the condition for the finite horizon near optimal approximate turnpike property in Definition 4.1 and thus (4.1) yields the inequality

$$
\#\left\{k \in\{0, \ldots, M\} \mid d\left(x(k), x^{e}\right) \geq \varepsilon\right\} \leq C_{\varepsilon, \mathbb{X}_{b}}^{\text {fin }}
$$

for all sufficiently large $N \in \mathbb{N}$. From this we obtain the infinite horizon near optimal approximate turnpike property (4.2) with $C_{\varepsilon, \mathbb{X}_{b}}^{\infty}=C_{\varepsilon, \mathbb{X}_{b}}^{f i n}$ and $\delta_{\varepsilon, M, \mathbb{X}_{b}}^{\infty}=\delta$.

"Definition $4.2 \Rightarrow$ Definition 4.1": Similar to the second part of the proof of Theorem 3.4, we consider a trajectory satisfying the conditions of Definition 4.1 from which we construct an extended trajectory satisfying the conditions of Definition 4.2. As in the first part of the proof, we need to take care of the bounds of $\delta$ in these definitions.

Fix again $\varepsilon>0$, a bounded set $\mathbb{X}_{b} \subseteq \mathbb{X}$ and $M \in \mathbb{N}$ and let $\delta^{\infty}=\delta_{\varepsilon, M, \mathbb{X}_{b}}^{\infty}>0$ be the level of accuracy needed in Definition 4.2. We set $\delta:=\delta^{\infty} / 8$ and pick $N_{0} \in \mathbb{N}$ from Assumption 4.3(ii). From Assumption 4.3(i) we can find $N_{1} \geq N_{0}$ such that $\left|V_{N^{\prime}}\left(x_{0}\right)-V_{N}\left(x_{0}\right)\right| \leq \delta$ and $\left|V_{N^{\prime}}\left(x_{0}\right)-V_{\infty}\left(x_{0}\right)\right| \leq \delta$ for all $x_{0} \in \mathbb{X}_{b}$ and all $N, N^{\prime} \geq N_{1}$. Moreover, we may pick $N_{1}$ so large that $\tilde{\varepsilon}$ from Assumption 4.3(ii) satisfies $\tilde{\varepsilon}<\delta^{\infty} / 4$. Finally, we set $N^{\prime}=\max \left\{M, N_{1}\right\}$.

Then, for arbitrary $N \geq N^{\prime}$ we pick a control sequence satisfying the conditions of Definition 4.1, i.e., with $J_{N}\left(x_{0}, u\right) \leq V_{N}\left(x_{0}\right)+\delta$. This implies

$$
\begin{aligned}
V_{N^{\prime}}\left(x_{0}\right)+2 \delta & \geq V_{N}\left(x_{0}\right)+\delta \geq J_{N}\left(x_{0}, u\right)=J_{N^{\prime}}\left(x_{0}, u\right)+\beta^{N^{\prime}} J_{N-N^{\prime}}\left(x\left(N^{\prime}\right), u\left(N^{\prime}+\cdot\right)\right) \\
& \geq J_{N^{\prime}}\left(x_{0}, u\right)+\beta^{N^{\prime}} V_{N-N^{\prime}}\left(x\left(N^{\prime}\right)\right) \geq J_{N^{\prime}}\left(x_{0}, u\right)-\tilde{\varepsilon},
\end{aligned}
$$

and thus $J_{N^{\prime}}\left(x_{0}, u\right) \leq V_{N^{\prime}}\left(x_{0}\right)+2 \delta+\tilde{\varepsilon}$. Picking another control sequence $\tilde{u}$ satisfying $J_{\infty}\left(x\left(N^{\prime}\right), \tilde{u}\right) \leq V_{\infty}\left(x\left(N^{\prime}\right)\right)+\delta$ and defining

$$
\hat{u}(k):= \begin{cases}u(k), & k=0, \ldots, N^{\prime}-1 \\ \tilde{u}\left(k-N^{\prime}\right), & k=N^{\prime}, N^{\prime}+1, \ldots\end{cases}
$$

we thus obtain

$$
\begin{aligned}
J_{\infty}\left(x_{0}, \hat{u}\right) & =J_{N^{\prime}}\left(x_{0}, u\right)+\beta^{N^{\prime}} J_{\infty}\left(x\left(N^{\prime}\right), \tilde{u}\right) \leq V_{N^{\prime}}\left(x_{0}\right)+2 \delta+\tilde{\varepsilon}+\beta^{N^{\prime}} V_{\infty}\left(x\left(N^{\prime}\right)\right)+\delta \\
& \leq V_{\infty}\left(x_{0}\right)+4 \delta+2 \tilde{\varepsilon}=V_{\infty}\left(x_{0}\right)+\delta^{\infty} .
\end{aligned}
$$

Hence, the extended trajectory satisfies the condition of Definition 4.2 and thus (4.2) yields

$$
\#\left\{k \in\{0, \ldots, M\} \mid d\left(x(k), x^{e}\right) \geq \varepsilon\right\} \leq C_{\varepsilon, \mathbb{X}_{b}}^{\infty} .
$$

This implies (4.1) and thus the finite horizon near optimal approximate turnpike property from Definition 4.2 for $N \geq N^{\prime}$ with $C_{\varepsilon, \mathbb{X}_{b}}^{f i n}=C_{\varepsilon, \mathbb{X}_{b}}^{\infty}$ and $\delta_{\varepsilon, M, \mathbb{X}_{b}}^{f i n}=\delta$. For arbitrary $N$ we thus obtain (4.1) with $C_{\varepsilon, \mathbb{X}_{b}}^{\text {fin }}=\max \left\{N^{\prime}, C_{\varepsilon, \mathbb{X}_{b}}^{\infty}\right\}$. 


\section{Turnpike with transient estimates}

As already mentioned, the turnpike definitions so far do not allow for estimating how fast the trajectories approach the equilibrium $x^{e}$. They also do not allow for bounds on the trajectories during the time in which they are not close to $x^{e}$. In this section, we propose definitions for finite and infinite horizon turnpike properties that provide this information. Here, the infinite horizon definition was inspired by the usual notion of asymptotic stability (in its formulation via $\mathcal{K} \mathcal{L}$-functions which has become standard in nonlinear control, see [9]), while the finite horizon definition can be seen as an extension of the exponential turnpike property established in [3] under a strict dissipativity condition. Like in the previous sections, we will then be able to show that these two conditions are equivalent under suitable regularity conditions on the optimal control problem (2.1). In order to streamline the presentation, we limit ourselves to a set of assumptions suitable for the undiscounted setting from Section 3, i.e., to $\beta=1$.

For the following definitions, we recall that $\mathcal{K}$ is the space of functions $\alpha: \mathbb{R}_{0}^{+} \rightarrow \mathbb{R}_{0}^{+}$which are continuous and strictly increasing with $\alpha(0)=0$ and that $\mathcal{K} \mathcal{L}$ is the space of functions $\phi: \mathbb{R}_{0}^{+} \times \mathbb{R}_{0}^{+} \rightarrow \mathbb{R}_{0}^{+}$which are continuous, $r \mapsto \phi(r, t)$ is a $\mathcal{K}$-function for each $t \geq 0$ and $t \mapsto \phi(r, t)$ is strictly decreasing to 0 for each $r>0$. The space $\mathcal{L}_{\mathbb{N}_{0}}$ denotes all functions $\gamma: \mathbb{N}_{0} \rightarrow \mathbb{R}_{0}^{+}$which are strictly decreasing to 0 .

Definition 5.1: (finite horizon) The optimal control problem (2.1) has the finite horizon robust $\mathcal{K} \mathcal{L}$-turnpike property at an equilibrium $x^{e} \in \mathbb{X}$, if for each bounded set $\mathbb{X}_{b} \subset \mathbb{X}$ there are $\phi \in \mathcal{K} \mathcal{L}, \omega \in \mathcal{K}$ and $\gamma \in \mathcal{L}_{N_{0}}$ such that for each $\delta>0, N \in \mathbb{N}$ and all trajectories $(x(k), u(k))$ with $x_{0} \in \mathbb{X}_{b}, u(\cdot) \in \mathbb{U}^{N}\left(x_{0}\right)$ and satisfying $J_{N}\left(x_{0}, u\right) \leq V_{N}\left(x_{0}\right)+\delta$ the inequality

$$
d\left(x(k), x^{e}\right) \leq \phi\left(d\left(x_{0}, x^{e}\right), k\right)+\omega(\delta+\gamma(N)+\gamma(N-j))
$$

holds for all $j=0, \ldots, N$ and all $k=0, \ldots, j$.

Definition 5.2: (infinite horizon) The optimal control problem (2.1) has the infinite horizon robust $\mathcal{K} \mathcal{L}$-turnpike property at an equilibrium $x^{e} \in \mathbb{X}$, if for each bounded set $\mathbb{X}_{b} \subset \mathbb{X}$ there are $\phi \in \mathcal{K} \mathcal{L}$ and $\omega \in \mathcal{K}$ such that for each $\delta>0$ and all trajectories $(x(k), u(k))$ with $x_{0} \in \mathbb{X}_{b}, u(\cdot) \in \mathbb{U}^{\infty}\left(x_{0}\right)$ and satisfying $J_{\infty}\left(x_{0}, u\right) \leq V_{\infty}\left(x_{0}\right)+\delta$ the inequality

$$
d\left(x(k), x^{e}\right) \leq \phi\left(d\left(x_{0}, x^{e}\right), k\right)+\omega(\delta)
$$

holds.

We note that the second inequality implies that optimal trajectories $x^{\star}(k)$ starting at $x=x^{e}$ satisfy $x^{\star}(k)=x^{e}$. Hence, in order to ensure that $V_{\infty}\left(x^{e}\right)$ is finite, we need that $\min _{u \in \mathbb{U}, f\left(x^{e}, u\right)=x^{e}} \ell\left(x^{e}, u\right)=0$, which implies $V_{\infty}\left(x^{e}\right)=0$. We may thus assume $V_{\infty}\left(x^{e}\right)=0$ without loss of generality in the remainder of this section. We note that this assumption does not imply $V_{N}\left(x^{e}\right) \approx 0$, even for large $N$.

In order to show equivalence of Definitions 5.1 and 5.2, in addition to Assumption 3.3 we need the following assumption.

Assumption 5.3: For the optimal control problem (2.1) we assume that there is $K \in \mathbb{R}$ such that for any bounded set $\mathbb{X}_{b} \subseteq \mathbb{X}$ there is $\rho \in \mathcal{L}_{\mathbb{N}_{0}}$ such that for all $x \in \mathbb{X}_{b}$ the 
inequality

$$
\left|V_{\infty}(x)-V_{N}(x)+K\right| \leq \rho(N)
$$

holds

The intuition behind Assumption 5.3 is the following: assume the infinite horizon problem has an optimal equilibrium $\left(x^{e}, u^{e}\right)$ with $\ell\left(x^{e}, u^{e}\right)=0$. Then we have $V_{\infty}\left(x^{e}\right)=0$, but since on finite horizons $\left(x^{e}, u^{e}\right)$ will typically not be an optimal equilibrium, in general $\lim _{N \rightarrow \infty} V_{N}\left(x^{e}\right)=0$ will not hold. In this case, this limit value is the candidate for the value $K$ for which Assumption 5.3 holds. The following lemma shows that this reasoning can be made precise under rather mild conditions if the turnpike property holds.

Lemma 5.4: Consider the optimal control problem (2.1) and assume that the problem exhibits the turnpike property according to Definitions 3.1 and 3.2 and $V_{\infty}\left(x^{e}\right)=0$. Assume moreover that the $\operatorname{limit}_{\lim _{N \rightarrow \infty}} V_{N}\left(x^{e}\right)$ exists and that the optimal value functions $V_{N}$ are continuous at $x^{e}$ uniformly in $N \in \mathbb{N} \cup\{\infty\}$ in the following way: there exists $\sigma \in \mathcal{K}$ and $\nu \in \mathcal{L}_{\mathbb{N}_{0}}$ such that the inequality

$$
\left|V_{N}(x)-V_{N}\left(x^{e}\right)\right| \leq \sigma\left(d\left(x, x^{e}\right)\right)+\nu(N)
$$

holds for all $x \in \mathbb{X}$ and $N \in \mathbb{N} \cup\{\infty\}$, with the convention $\nu(\infty)=0$. Then Assumption 5.3 is satisfied.

Proof. We show that the assertion follows for $K=\lim _{N \rightarrow \infty} V_{N}\left(x^{e}\right)$. We choose $\eta \in \mathcal{L}_{\mathbb{N}_{0}}$ such that $\left|V_{N}\left(x^{e}\right)-K\right| \leq \eta(N)$ for all $N \in \mathbb{N}$ and fix a bounded set $\mathbb{X}_{b} \subseteq \mathbb{X}$. Moreover, we note that it is sufficient to prove the assertion for sufficiently large $N$, because the continuity assumption implies boundedness of $V_{N}$ and $V_{\infty}$ on bounded sets, which ensures existence of $\rho(N)$ for finitely many $N$.

We first show that there exists $\rho_{1} \in \mathcal{L}_{\mathbb{N}_{0}}$ such that $V_{\infty}(x) \leq V_{N}\left(x^{e}\right)-K+\rho_{1}(N)$ holds for all $x \in \mathbb{X}_{b}$. To this end, fix $\delta_{0}>0$, let $\delta \in\left(0, \delta_{0}\right), x \in \mathbb{X}_{b}$ and consider a control $u^{\delta}$ with $J_{N}\left(x, u^{\delta}\right) \leq V_{N}(x)+\delta$. Then, for sufficiently large $N \in \mathbb{N}$ and $\varepsilon>0$ the constant $C_{\delta_{0}, \varepsilon, \mathbb{X}_{b}}^{\text {fin }}$ from Definition 3.1 satisfies $C_{\delta_{0}, \varepsilon, \mathbb{X}_{b}}^{\text {fin }} \geq N / 2$. We define $\varepsilon=\varepsilon(N)>0$ minimal such that this inequality holds. Then, since for each $\varepsilon>0$ there is $N \in \mathbb{N}$ such that $C_{\delta_{0}, \varepsilon, \mathbb{X}_{b}}^{\text {fin }} \geq N / 2$ holds, it follows that $\varepsilon(N) \rightarrow 0$ as $N \rightarrow \infty$. Hence, there is $\tilde{\varepsilon}(\cdot) \in \mathcal{L}_{\mathbb{N}_{0}}$ with $\varepsilon(N) \leq \tilde{\varepsilon}(N)$, e.g., $\tilde{\varepsilon}(N)=\sup _{K \geq N} \varepsilon(K)+2^{-N}$. For each $N$ we now pick the minimal $k^{*} \in\{0, \ldots, N\}$ satisfying $d\left(x_{u^{\delta}}\left(k^{*}\right), x^{e}\right)<\varepsilon(N)$, which because of $C_{\delta_{0}, \varepsilon, \mathbb{X}_{b}}^{\text {fin }} \geq N / 2$ satisfies $N-k^{*} \geq\lfloor N / 2\rfloor$. We pick a control $\hat{u}^{\delta}$ satisfying $J_{\infty}\left(x_{u^{\delta}}\left(k^{*}\right), \hat{u}^{\delta}\right) \leq V_{\infty}\left(x_{u^{\delta}}\left(k^{*}\right)\right)+\delta$ and set $u(k)=u^{\delta}(k)$, $k=0, \ldots, k^{*}-1$ and $u(k)=\hat{u}^{\delta}\left(k+k^{*}\right), k \geq k^{*}$. Then we can estimate

$$
\begin{aligned}
V_{\infty}(x) \leq J_{\infty}(x, u) & =J_{k^{*}}\left(x, u^{\delta}\right)+J_{\infty}\left(x_{u^{\delta}}\left(k^{*}\right), \hat{u}^{\delta}\right) \\
& \leq J_{N}\left(x, u^{\delta}\right)-J_{N-k^{*}}\left(x_{u^{\delta}}\left(k^{*}\right), u^{\delta}\left(k^{*}+\cdot\right)\right)+V_{\infty}\left(x_{u^{\delta}}\left(k^{*}\right)\right)+\delta \\
& \leq V_{N}(x)+\delta-V_{N-k^{*}}\left(x_{u^{\delta}}\left(k^{*}\right)\right)+V_{\infty}\left(x_{u^{\delta}}\left(k^{*}\right)\right)+\delta \\
& \leq V_{N}(x)-K+V_{\infty}\left(x^{e}\right)+2 \sigma(\varepsilon(N))+\nu\left(N-k^{*}\right)+\eta(N)+2 \delta .
\end{aligned}
$$

Since $\delta>0$ was arbitrary, $N-k^{*} \geq\lfloor N / 2\rfloor$ and $V_{\infty}\left(x^{e}\right)=0$, this shows the claim with $\rho_{1}(N)=2 \sigma(\tilde{\varepsilon}(N))+\nu(\lfloor N / 2\rfloor)+\eta(N)$. 
The converse inequality $V_{N}(x) \leq V_{\infty}\left(x^{e}\right)+K+\rho_{2}(N)$ is obtained similarly, starting from a $\delta$-optimal trajectory for the $\infty$-horizon problem and extending it after the "turnpike time" $k^{*}$ by a $\delta$-optimal trajectory for the problem with horizon $N-k^{*}$. Together this yields the assertion with $\rho=\max \left\{\rho_{1}, \rho_{2}\right\}$.

The first equivalence theorem for Definitions 5.1 and 5.2 now uses Assumption 5.3.

Theorem 5.5: Consider the optimal control problem (2.1) and assume that

(i) $\left|V_{\infty}\right|$ is bounded on bounded subsets of $\mathbb{X}$

(ii) Assumption 5.3 holds

(iii) for each $\Theta>0$ there is a bounded set $\mathbb{X}_{\Theta} \subseteq \mathbb{X}$ such that for each $N \in \mathbb{N} \cup\{\infty\}$ the inequality $J_{N}\left(x_{0}, u\right) \leq \Theta$ implies $x(k) \in \mathbb{X}_{\Theta}$ for all $k=0, \ldots, N$.

Then Definition 5.1 holds if and only if Definition 5.2 holds.

Proof. "Definition $5.1 \Rightarrow$ Definition 5.2": Consider a trajectory $x(\cdot)$ with control $u(\cdot)$ and initial value $x_{0}$ satisfying the conditions of Definition 5.2. Then for all $j \in \mathbb{N}$ we obtain

$$
J_{j}\left(x_{0}, u\right)+V_{\infty}(x(j)) \leq J_{\infty}\left(x_{0}, u\right) \leq V_{\infty}\left(x_{0}\right)+\delta .
$$

Then from (ii) with $\mathbb{X}_{b}=\mathbb{X}_{\Theta}$ from (iii), for arbitrary $N \in \mathbb{N}$ with $j \leq N$ we obtain

$$
J_{j}\left(x_{0}, u\right) \leq V_{\infty}\left(x_{0}\right)-V_{\infty}(x(j))+\delta \leq V_{N}\left(x_{0}\right)-V_{N-j}(x(j))+\delta+\rho(N)+\rho(N-j) .
$$

Now taking the control $u(k)$ for $k=0, \ldots, j-1$ and extending it with an $\varepsilon$-optimal control for horizon $N-k$, arbitrary $\varepsilon>0$ and initial value $x(j)$ yields a control $\tilde{u}$ satisfying

$$
J_{N}\left(x_{0}, \tilde{u}\right) \leq J_{j}\left(x_{0}, u\right)+V_{N-j}(x(j))+\varepsilon \leq V_{N}\left(x_{0}\right)+\delta+\rho(N)+\rho(N-j)+\varepsilon .
$$

Hence, Definition 5.1 with $\delta+\rho(N)+\rho(N+j)+\varepsilon$ in place of $\delta$ implies the estimate

$$
d\left(x(k), x^{e}\right) \leq \phi\left(d\left(x_{0}, x^{e}\right), k\right)+\omega(\delta+\rho(N)+\gamma(N)+\rho(N-j)+\gamma(N-j)+\varepsilon)
$$

for all $k=0, \ldots, j$. Fixing $k$ and letting $\varepsilon \rightarrow 0, N \rightarrow \infty$ and $j:=\lfloor N / 2\rfloor \rightarrow \infty$, continuity of $\phi$ and $\omega$ and the fact that $\rho \in \mathcal{L}_{\mathbb{N}_{0}}$ and $\gamma \in \mathcal{L}_{\mathbb{N}_{0}}$ yield the desired inequality

$$
d\left(x(k), x^{e}\right) \leq \phi\left(d\left(x_{0}, x^{e}\right), k\right)+\omega(\delta) .
$$

"Definition $5.2 \Rightarrow$ Definition 5.1": Consider a trajectory $x(\cdot)$ of length $N$ with control $u(\cdot)$ and initial value $x_{0}$ satisfying the conditions of Definition 5.1. Then for all $j=0, \ldots, N$ we obtain

$$
J_{j}\left(x_{0}, u\right)+V_{N-j}(x(j)) \leq J_{N}\left(x_{0}, u\right) \leq V_{N}\left(x_{0}\right)+\delta .
$$

Then from (ii) with $\mathbb{X}_{b}=\mathbb{X}_{\Theta}$ from (iii) we obtain

$$
J_{j}\left(x_{0}, u\right) \leq V_{N}\left(x_{0}\right)-V_{N-j}(x(j))+\delta \leq V_{\infty}\left(x_{0}\right)-V_{\infty}(x(j))+\delta+\rho(N)+\rho(N-j) .
$$


Now taking the control $u(k)$ for $k=0, \ldots, j-1$ and extending it with an $\varepsilon$-optimal control for infinite horizon for arbitrary $\varepsilon>0$ and initial value $x(j)$ yields a control $\tilde{u}$ satisfying

$$
J_{\infty}\left(x_{0}, \tilde{u}\right) \leq J_{j}\left(x_{0}, u\right)+V_{\infty}(x(j))+\varepsilon \leq V_{\infty}\left(x_{0}\right)+\delta+\rho(N)+\rho(N-j)+\varepsilon .
$$

Hence, using Definition 5.2 with $\delta+\rho(N)+\rho(N+j)+\varepsilon$ in place of $\delta$ yields the estimate

$$
d\left(x(k), x^{e}\right) \leq \phi\left(d\left(x_{0}, x^{e}\right), k\right)+\omega(\delta+\rho(N)+\rho(N-j)+\varepsilon)
$$

for all $k=0, \ldots, j$. For $\varepsilon \rightarrow 0$, continuity of $\omega$ yields the desired inequality for $\gamma=\rho \in$ $\mathcal{L}_{\mathbb{N}_{0}}$.

Using Lemma 5.4 we can obtain a variant of Theorem 5.5 avoiding the use of Assumption 5.3.

Corollary 5.6: Consider the optimal control problem (2.1) and assume that

(i) $V_{\infty}\left(x^{e}\right)=0$ and $\lim _{N \rightarrow \infty} V_{N}\left(x^{e}\right)$ exists

(ii) the optimal value functions $V_{N}$ are continuous at $x^{e}$ uniformly in $N \in \mathbb{N} \cup\{\infty\}$ in the following way: there exists $\gamma \in \mathcal{K}$ and $\nu \in \mathcal{L}_{\mathbb{N}_{0}}$ such that the inequality

$$
\left|V_{N}(x)-V_{N}\left(x^{e}\right)\right| \leq \gamma\left(d\left(x, x^{e}\right)\right)+\nu(N)
$$

holds for all $x \in \mathbb{X}$ and $N \in \mathbb{N} \cup\{\infty\}$ with the convention $\nu(\infty)=0$.

(iii) for each $\Theta>0$ there is a bounded set $\mathbb{X}_{\Theta} \subseteq \mathbb{X}$ such that for each $N \in \mathbb{N} \cup\{\infty\}$ the inequality $J_{N}\left(x_{0}, u\right) \leq \Theta$ implies $x(k) \in \mathbb{X}_{\Theta}$ for all $k=0, \ldots, N$.

Then Definition 5.1 holds if and only if Definition 5.2 holds.

Proof. We note that (i) and (ii) imply boundedness of $V_{\infty}$ and $V_{N}$ on bounded sets. Moreover we note that the turnpike property from Definition 5.1 implies that of Definition 3.1 and that the property from Definition 5.2 implies that of Definition 3.2. Since by Theorem 3.4 the properties from Definitions 3.1 and 3.2 are equivalent under the conditions of the corollary, we obtain that if either Definition 5.1 or Definition 5.2 holds, then both Definitions 3.1 and 3.2 follow. Hence we can apply Lemma 5.4 in order to conclude that Assumption 5.3 holds. The assertion then follows from Theorem 5.5.

We illustrate the use of Theorem 5.5 by the following well known class of optimal control problems.

Example 5.7: Consider an undiscounted linear quadratic optimal control problem with

$$
f(x, u)=A x+B u \quad \text { and } \quad \ell(x, u)=\left(x^{T}, u^{T}\right) G\left(\begin{array}{l}
x \\
u
\end{array}\right),
$$

where $(A, B)$ is stabilizable and the matrix $G$ is symmetric and positive definite. It is well known that for such a problem the optimal trajectories converge to the origin exponentially fast and that the infinite horizon optimal value function is of the form $V_{\infty}(x)=x^{T} Q_{\infty} x$ for a symmetric and positive definite matrix $Q_{\infty}$. Moreover, the optimal control is available 
in linear feedback form, i.e., $u^{*}=F x$ and $V_{\infty}$ is a quadratic Lyapunov function. More precisely the inequality

$$
V(A x+B F x) \leq V(x)-\ell(x, F x)
$$

holds for all $x \in \mathbb{R}^{n}$. For all trajectories $(x(k), u(k))$ satisfying $J_{\infty}\left(x_{0}, u\right) \leq V_{\infty}\left(x_{0}\right)+\delta$ and all $k \in \mathbb{N}$ it holds that $J_{\infty}(x(k), u(k+\cdot)) \leq V_{\infty}(x(k))+\delta$ which implies

$$
\begin{aligned}
V_{\infty}(x(k+1)) & \leq J_{\infty}(x(k+1), u(k+1+\cdot))=J_{\infty}(x(k), u(k+\cdot))-\ell(x(k), u(k)) \\
& \leq V_{\infty}(x(k))-\ell(x(k), u(k))+\delta .
\end{aligned}
$$

From this inequality a standard Lyapunov argument yields the existence of $C_{1}, C_{2}>0$ and $a \in(0,1)$ such that

$$
V_{\infty}(x(k)) \leq \max \left\{C_{1} a^{k} V_{\infty}(x(0)), C_{2} \delta\right\}
$$

for all $k \in \mathbb{N}$, which implies

$$
\|x(k)\| \leq \phi(\|x(0)\|, k)+\omega(\delta)
$$

with $\phi(r, k)=C \sqrt{a}^{k} r$ and $\omega(r)=C \sqrt{\delta}$ for an appropriate constant $C>0$. This implies that the problem has the infinite horizon robust $\mathcal{K} \mathcal{L}$-turnpike property according to Definition 5.2.

In order to show that the problem also has the finite horizon robust $\mathcal{K} \mathcal{L}$-turnpike property according to Definition 5.1, we now check that the problem satisfies the conditions of Theorem 5.5. Condition (i) is obviously satisfied, since $V_{\infty}$ is a quadratic function. Condition (ii) follows with $K=0$ from the fact that $V_{N}(x)=x^{T} Q_{N} x$ and the matrices $Q_{N}$ are defined via the Riccati difference equation and converge exponentially fast towards $Q_{\infty}$, which is the solution of the discrete time algebraic Riccati equation. The exponential convergence moreover implies that $\rho$ can be chosen to be of the form $\rho(N)=D b^{N}$ with $D>0$ and $b \in(0,1)$. Condition (iii) follows immediately from the fact that $G$ is positive definite, implying the existence of $C_{\Theta}>0$ such that $\ell(x, u)>\Theta$ whenever $\|x\| \geq C_{\Theta}$. From this condition (iii) follows with $\mathbb{X}_{\Theta}=\left\{x \in \mathbb{R}^{n} \mid\|x\| \leq C_{\Theta}\right\}$.

Thus, all conditions of Theorem 5.5 hold and we can conclude the finite horizon $\mathcal{K} \mathcal{L}$ turnpike property with $\left.\phi(r, k)=C \sqrt{a}^{k} r, \omega(r)=C \sqrt{(} r\right)$ and $\rho(N)=D b^{N}$, i.e., both $\phi$ and $\rho$ are exponentially decaying in time $k$ or in the horizon $N$, respectively.

\section{Conclusions}

In this paper, we have investigated the relationship between turnpike properties for finite and infinite horizon optimal control problems with the same stage cost. Specifically, we have shown that under mild technical assumptions, these properties are equivalent. Furthermore, this relationship has been demonstrated for optimal control problems involving both undiscounted and discounted stage costs, making the results applicable to commonly studied problems in both engineering and mathematical economics.

Furthermore, we have proposed a definition of a turnpike property that incorporates information about rate of convergence for optimal trajectories approaching an optimal equilibria, as well as a bound on how far such trajectories can be from this equilibria during 
the time when they are not close. This robust $\mathcal{K} \mathcal{L}$-turnpike property provides a potential route to better quantitative results in problems involving turnpikes, similar to the modern use of comparison functions in stability theory.

\section{References}

[1] B. D. O. Anderson And P. V. Kokotović, Optimal control problems over large time intervals, Automatica, 23 (1987), pp. 355-363.

[2] D. Angeli, R. Amrit, And J. B. Rawlings, On average performance and stability of economic model predictive control, IEEE Trans. Autom. Control, 57 (2012), pp. 1615-1626.

[3] T. Damm, L. Grüne, M. Stieler, And K. Worthmann, An exponential turnpike theorem for dissipative discrete time optimal control problems, SIAM J. Control Optim., 52 (2014), pp. 1935-1957.

[4] R. Dorfman, P. A. Samuelson, And R. M. Solow, Linear Programming and Economic Analysis, Dover Publications, New York, 1987. Reprint of the 1958 original.

[5] T. Faulwasser, M. Korda, C. N. Jones, and D. Bonvin, Turnpike and dissipativity properties in dynamic real-time optimization and economic $M P C$, in Proceedings of the 53rd IEEE Conference on Decision and Control — CDC 2014, 2014, pp. 27342739 .

[6] L. GRÜNE, Economic receding horizon control without terminal constraints, Automatica, 49 (2013), pp. 725-734.

[7] L. GRüne, Approximation properties of receding horizon optimal control, Jahresber. DMV, 118 (2016), pp. 3-37.

[8] L. Grüne And M. A. Müller, On the relation between strict dissipativity and the turnpike property, Syst. Contr. Lett., 90 (2016), pp. 45-53.

[9] C. M. Kellett, A compendium of comparison function results, Math. Control Signals Systems, 26 (2014), pp. 339-374.

[10] L. W. MCKenzIE, Optimal economic growth, turnpike theorems and comparative dynamics, in Handbook of Mathematical Economics, Vol. III, vol. 1 of Handbooks in Econom., North-Holland, Amsterdam, 1986, pp. 1281-1355.

[11] A. Porretta And E. Zuazua, Long time versus steady state optimal control, SIAM J. Control Optim., 51 (2013), pp. 4242-4273.

[12] E. TRÉlat And E. Zuazua, The turnpike property in finite-dimensional nonlinear optimal control, J. Differential Equations, 258 (2015), pp. 81-114.

[13] J. von Neumann, A model of general economic equilibrium, The Review of Economic Studies, 13 (1945), pp. 1-9. 
[14] A. Zaslavski, Turnpike Phenomenon and Infinite Horizon Optimal Control, Springer International Publishing, 2014. 